\title{
Application of Modified Flower Pollination Algorithm to Multiple Vehicle Routing Problems with Time Constraints
}

\author{
SUPAPORN SUWANNARONGSRI \\ Department of Materials Handling and Logistics Engineering, Faculty of Engineering, \\ King Mongkut's University of Technology North Bangkok \\ 1518 Pracharaj-1 Rd., Bangsue, Bangkok, 10800 \\ THAILAND
}

\begin{abstract}
The aim of this research is to apply the modified flower pollination algorithm (MoFPA) to the multiple vehicle routing problems (MVRP) with the time constraints. As one of the latest enhanced versions of the original flower pollination algorithm (FPA), the MoFPA utilizes the randomly switching probability for selection between local (self) and global (cross) pollinations in order to search for the better solutions and improve its search performance. In this paper, the MoFPA is applied to solve the MVRP problems with the time constraints based on the modern optimization approach. Ten standard MVRP problems with the time constraints consisting of approximately 100-500 destinations are selected and solved by the MoFPA. Results obtained by the MoFPA are compared with those obtained by original FPA, genetic algorithm (GA), tabu search (TS) and particle swarm optimization (PSO). Results reveals that the MoFPA can provide optimal solutions of all ten selected MVRP problems with shorter total distance than FPA, PSO, TS and GA, respectively. In addition, the execution time consumed by FPA and MoFPA are less than PSO, TS and GA, respectively.
\end{abstract}

Key-Words: - Modified flower pollination algorithm, Randomly switching probability, Multiple vehicle routing problem, Time constraints, Metaheuristic optimization technique, Modern optimization

Received: February 16, 2020. Revised: August 30, 2020. Accepted: September 10, 2020. Published: September 28, 2020.

\section{Introduction}

In 2012, the flower pollination algorithm (FPA) was firstly proposed by Yang as the population-based metahueristic optimization search technique for solving both continuous and combinatorial, singleobjective and multi-objective optimization problems [1]. The FPA algorithm mimics the pollination behaviour of flowering plants in nature. The FPA algorithm was proved for the global convergent property [2]. Since 2012, the FPA has shown superiority to other popular metaheuristic algorithms including the genetic algorithm (GA) and particle swarm optimization (PSO) [1-3]. From literature review, the FPA has been successfully applied to solve various real-world engineering problems, such as economic/emission dispatch, reactive power dispatch, optimal power flow, solar PV parameter estimation, load frequency control, wireless sensor networks, linear antenna array optimization, frames and truss systems, structure engineering design, multilevel image thresholding, travelling transportation problem, control system design and model identification. Development and significant applications of the FPA have been reviewed and reported $[4,5]$.
Recently, many variants of FPA algorithms have been developed by modification, hybridization and cooperation manners in order to enhance its search performance for the complex optimization problems [5]. One of the modified flower pollination algorithms called the MFPA [6]. The MFPA hybridized the original FPA with the clonal selection algorithm (CSA) in order for generating some elite solutions. The binary flower pollination algorithm (BFPA) was developed for solving discrete and combinatorial optimization problems [7]. Another significant modification was proposed as the modified global FPA (mgFPA) [8]. The mgFPA was developed to utilize features of feasible solutions by extracting its characteristics, and utilize the exploration process to search for the optimal solution in specific search spaces [8]. One of the latest enhanced versions of the FPA is the modified flower pollination algorithm (MoFPA) proposed in 2020 by Pringsakul and Puangdownreong [9]. In the MoFPA algorithm, the switching probability for selection between local and global pollinations is changed from the fixed manner used in the original FPA to the random manner according to the pollination behaviour of flowering plants in nature. The MoFPA 
was tested against several standard benchmark functions for global minimization. The superiority of the MoFPA to the original FPA for function optimization has been reported [9]. Moreover, the MoFPA has been completely applied to design the proportional-integral-derivative-accelerated (PIDA) controller for the temperature control system [10].

In this paper, the MoFPA $[9,10]$ is applied to solve the multiple vehicle routing problems (MVRP) with the time constraints based on the modern optimization in order to find the set of routes with the shortest total distance for overall minimum cost serving all the given demands by the fleet of vehicles. The usefulness of the study can be utilized in practical applicability for any real-world MVRP problem. This paper consists of six sections. After an introduction is given in section 1, the original FPA and MoFPA algorithms are illustrated in section 2. The MVRP models and details of ten selected standard MVRP problems are described in section 3. Experimental results of the MoFPA-based MVRP problem optimization are performed in section 4 . Discussions are given in section 5. Finally, conclusions and future research are provided in section 6 .

\section{FPA and MoFPA Algorithms}

\subsection{FPA Algorithm}

As proposed by Yang in 2012 [1], the original FPA algorithm mimics the pollination behaviour of flowering plants in nature for survival and reproduction. The flower pollution in nature can be divided into two categories (self and cross pollinations) with two types of pollinator (biotic and abiotic pollinators) [11-13]. Self-pollination occurs from pollen gamete of the same flower or different flowers of the same plant. It usually occurs within short distance by abiotic pollinators such as wind and diffusion in water. Cross-pollination occurs from pollen gamete of the different flowers of different plants. It usually occurs at long distance by biotic pollinators such as insects and animals.

In the FPA algorithm, self-pollination with abiotic pollinators is regarded as the local pollination by using the random walk to search for the feasible solutions in local search spaces. With the local pollination, a new solution $\boldsymbol{x}_{i}$ (flower or pollen gamete) can be calculated by (1), where $\boldsymbol{x}_{j}$ and $\boldsymbol{x}_{k}$ are randomly selected solutions in the current iteration $t$ and $\varepsilon$ stands for a random with the uniform distribution that can be calculated by (2), where $a$ and $b$ are lower and upper bounds of random.

$$
\begin{gathered}
\boldsymbol{x}_{i}^{t+1}=\boldsymbol{x}_{i}^{t}+\varepsilon\left(\boldsymbol{x}_{j}^{t}-\boldsymbol{x}_{k}^{t}\right) \\
\varepsilon(\rho)= \begin{cases}1 /(b-a), & a \leq \rho \leq b \\
0, & \rho<a \text { or } \rho>b\end{cases}
\end{gathered}
$$

Cross-pollination with biotic pollinators is regarded as the global pollination by using the Lévyflight to search for the feasible solutions in global search space. With the global pollination, a new solution $\boldsymbol{x}_{i}$ can be calculated by (3), where $\boldsymbol{g}^{*}$ is the current best solution and $L$ is a random with the Lévyflight distribution that can be calculated by (4), where $\Gamma(\lambda)$ is the Gamma function as stated in (5).

$$
\begin{gathered}
\boldsymbol{x}_{i}^{t+1}=\boldsymbol{x}_{i}^{t}+L\left(\boldsymbol{x}_{i}^{t}-\boldsymbol{g}^{*}\right) \\
L \approx \frac{\lambda \Gamma(\lambda) \sin (\pi \lambda / 2)}{\pi} \frac{1}{s^{1+\lambda}}, \quad\left(s>>s_{0}>0\right) \\
\Gamma(\lambda)=\int_{0}^{\infty} t^{\lambda-1} e^{-t} d t
\end{gathered}
$$

In the original FPA algorithm, selection between local and global pollinations is controlled by the switch probability $p$. The values of $p$ are recommended to be fixed at any value between 0.15 0.25 [1-3]. The original FPA algorithm can be summarized by the pseudo code as shown in Fig. 1.

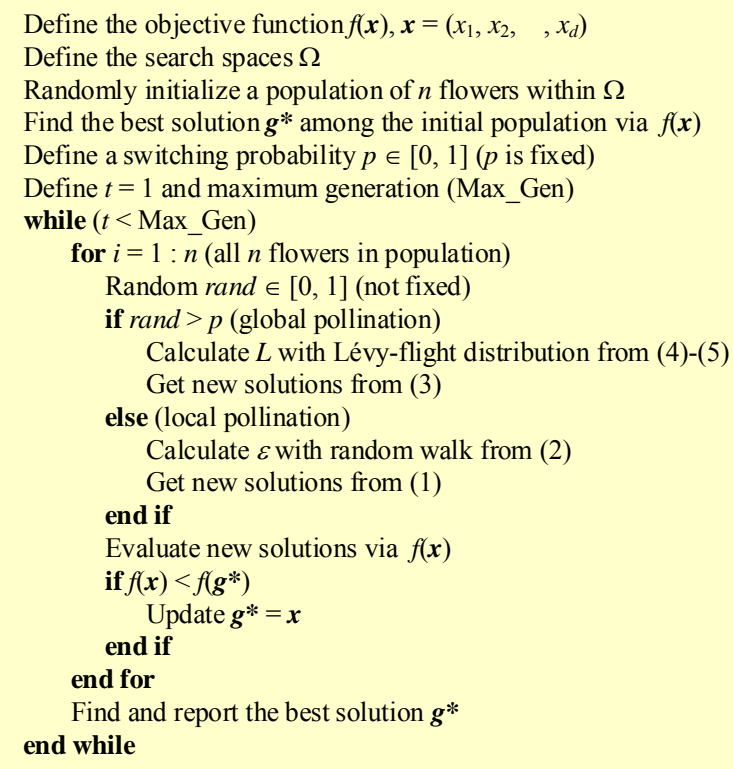

Fig. 1 Pseudo code of FPA algorithm.

\subsection{MoFPA Algorithm}

The MoFPA algorithm $[9,10]$ was proposed as one of the enhanced versions of the original FPA to improve its search performance. The MoFPA 
algorithm utilizes the randomly switching probability for selection between local and global pollinations. This leads the opportunity of the global finding according to the flower pollination behaviour in nature.

Regarding to the original FPA algorithm in Fig. 1, the condition of selection between local and global pollinations is rand $>p$, where rand $\in[0,1]$ is random number drawn from a uniform distribution and $p=0.20$ is a fixed value. If $r a n d>p$, the global pollination will be activated as shown in Fig. 2(a). Otherwise, the local pollination will be invoked. For the MoFPA algorithm, the condition of selection between local and global pollinations is changed to rand $>$ randp, where rand $\in[0,1]$ and randp $\in\left[p_{\text {min }}\right.$, $\left.p_{\max }\right]$ are random numbers drawn from a uniform distribution. With a new selecting condition, if rand $>$ randp, the global pollination will be activated as shown in Fig. 2(b). Otherwise, the local pollination will be invoked. The MoFPA algorithm can be represented by the pseudo code as shown in Fig. 3. The randp is recommended to be varied between $0.50-0.75[9,10]$. Although the modification in the MoFPA algorithm is very simple, it performs amazingly higher search performance than the original FPA $[9,10]$.

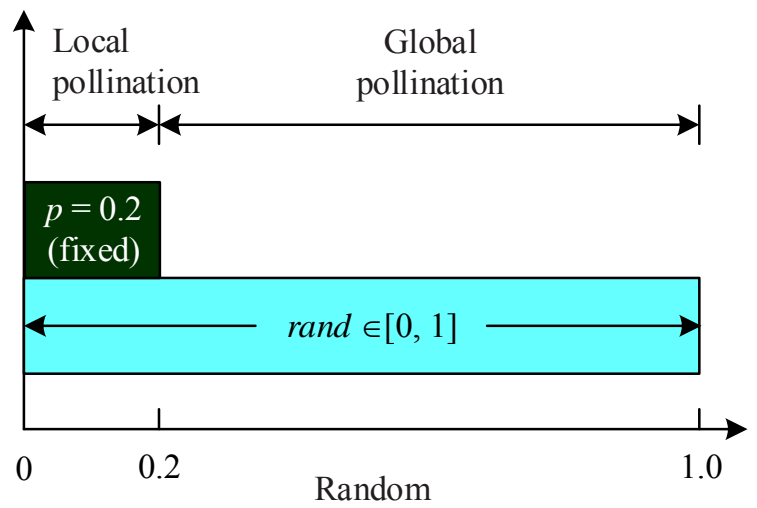

(a) FPA

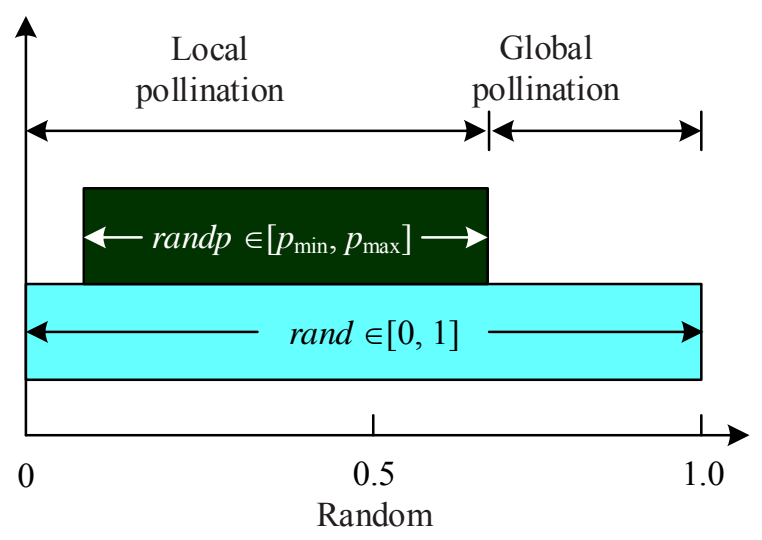

(b) MoFPA

Fig. 2 Selecting conditions between local and global pollinations of FPA and MoFPA.

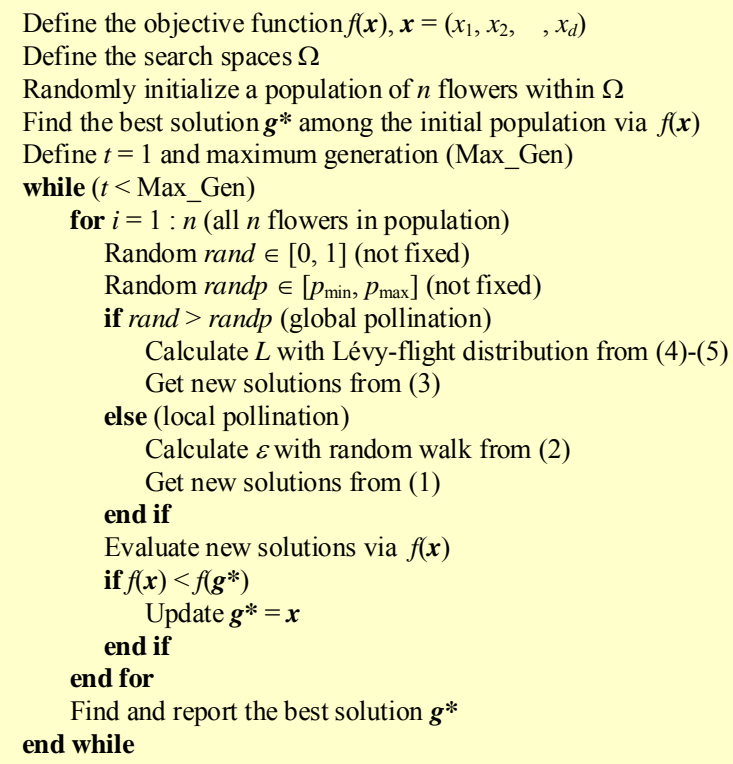

Fig. 3 Pseudo code of MoFPA algorithm.

\section{MVRP Problem Models}

\subsection{MVRP Models}

The MVRP model can be formulated based on the graph theory [14-20] as the multiple traveling salesman problem (MTSP). Generally, the MVRP consists of $n$ cities (nodes or destinations) and $m$ vehicles in a fleet. The distances between the $i$-th and $j$-th cities is represented by $c_{i j}$. In the symmetric case, $c_{i j}=c_{j i}$, for all cities $(i, j)$. They can be displayed by the distance metrix $d: n \times n \rightarrow \mathfrak{R}$. Assume that all vehicles will start at the home city (or depot). All vehicles in a fleet will take a route such that each city is visited by exactly one vehicle until all vehicles in the fleet return to the home city at the end of the tour. Based on the modern optimization, the MVRP model is then formulated as the objective function in (6), where $c(i, j)$ is the distances between the $i$-th and $j$-th cities and $\delta_{i, j, k}$ is the logical statement. If the vehicle $k$ can travels from any city $i$ to any another city $j, \delta_{i, j, k}$ $=1$. Otherwise, $\delta_{i, j, k}=0$.

Commonly, the MVRP problem model with time constraints possesses seven constrained functions as stated in (7)-(13).

- Constrained function-1 as expressed in (7) is used to ensure that all vehicles in a fleet will leave the home city exactly once.

- Constrained function-2 as stated in (8) is conducted to guarantees that all vehicles in a fleet will return to home city exactly once.

- Constrained function-3 as expressed in (9) is performed to ensure that all cities (except the home city) will be left from each vehicle in a fleet exactly once. 


$$
\begin{gathered}
\text { Minimize } \quad f_{\text {obj }}=\sum_{i=1}^{n} \sum_{j=1}^{n} \sum_{k=1}^{m} \delta_{i, j, k} c(i, j) \\
\text { Subject to } \quad \sum_{j=2}^{n} \delta_{1, j, k}=1, \quad \forall 1 \leq k \leq m \\
\qquad \sum_{i=2}^{n} \delta_{i, 1, k}=1, \quad \forall 1 \leq k \leq m \\
\sum_{j=1}^{n} \sum_{k=1}^{m} \delta_{i, j, k}=1, \quad \forall 2 \leq i \leq n \\
\sum_{i=1}^{n} \sum_{k=1}^{m} \delta_{i, j, k}=1, \quad \forall 2 \leq j \leq n \\
\sum_{i=1}^{n} \delta_{i, r, k}=\sum_{j=1}^{n} \delta_{r, j, k}, \forall 2 \leq r \leq n, \forall 1 \leq k \leq m \\
\sum_{i=1}^{n} \sum_{j=1}^{n} T_{i, j, k} \leq T_{\max }, \quad 1 \leq k \leq m \\
+(n-m) \cdot \sum_{k=1}^{m} \delta_{i, r, k} \leq n-m-1, \\
\quad \forall 2 \leq i \neq j \leq n
\end{gathered}
$$

- Constrained function-4 as stated in (10) is used to ensure that all vehicles in a fleet will return only once to each city (except the home city).

- Constrained function-5 as expressed in (11) is conducted to ensure that the number of times that a vehicle spends for visiting all cities (except the home city) equals the number of times that all cities are left.

- Constrained function-6 as stated in (12) is performed to ensure that no sub-tours exist (degenerate routes that do not include the home city), by using $n-1$ as dummy variables of $u_{2}, \ldots, u_{n}$.

- Constrained function-7 as expressed in (13) where $T_{i, j, k}$ is the traveling time of the vehicle $k$ from the $i$-th city to the $j$-th city and $T_{\max }$ is the maximum working time of each vehicle. This is the time constraints conducted to ensure that each vehicle in a fleet spends the working time within its defined maximum working time.

The objective of the MVRP problem in (6) associated with the constrained functions in (7)-(13) will be minimized by searching for the total traveling distances. In this work, only one home city and the symmetric case are assumed.

\subsection{Ten Selected MVRP Problems}

Recently, VRP and MVRP problems have been solved by the original FPA $[21,22]$. In this work, ten standard MVRP problems with the time constraints consisting of 100-500 destinations, approximately, from literatures $[23,24]$ are selected and solved by the MoFPA algorithm. Details of ten selected MVRP problems are summarized in Table 1. The city locations of MVRP\#1 (EIL101) are plotted in Fig. 4 to display their locations as an example, where $\square$ stands for the city locations.

Table 1 Ten selected MVRP problems [20, 21].

\begin{tabular}{ccccc}
\hline Problems & Names & $\begin{array}{c}\text { No. } \\
\text { of } \\
\text { Cities }\end{array}$ & $\begin{array}{c}\text { Optimal } \\
\text { Tour for } \\
\text { One } \\
\text { Vehicle } \\
\text { (Km.) }\end{array}$ & Comments \\
\hline MVRP\#1 & EIL101 & 101 & 629 & Eilon \\
MVRP\#2 & CH150 & 150 & 6,528 & Churritz \\
MVRP\#3 & BRG180 & 180 & 1,950 & Rinaldi \\
MVRP\#4 & D198 & 198 & 15,780 & Reinelt \\
MVRP\#5 & GIL262 & 262 & 2,378 & Gillet \\
MVRP\#6 & LIN318 & 318 & 42,029 & Kernighan \\
MVRP\#7 & GR431 & 431 & 171,414 & Groetschel \\
MVRP\#8 & PCB442 & 442 & 50,778 & Juenger \\
MVRP\#9 & ATT532 & 532 & 92,794 & Padberg \\
MVRP\#10 & PA561 & 561 & 2,763 & Kleinschmidt \\
\hline
\end{tabular}

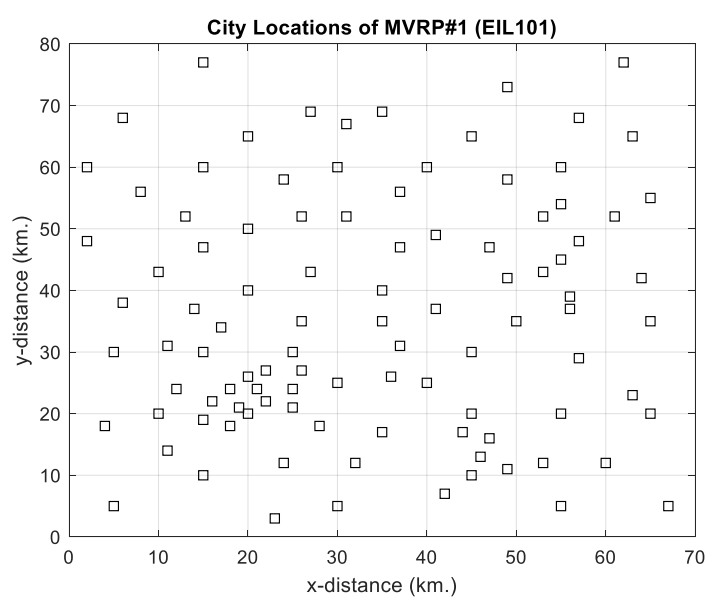

Fig. 4 City locations of MVRP\#1 (EIL101).

\section{Experimental Results}

Ten selected MVRP problems in Table 1 will be solved by the MoFPA algorithm compared with GA, TS, PSO and original FPA. Therefore, algorithms of GA, TS, PSO, FPA and MoFPA were coded by MATLAB version 2018 b run on Intel(R) Core(TM) 15-3470 CPU@3.60GHz. 50 trial-runs are executed for each MVRP problem to search for its best solution. In order to set the time constraint in (13), the daily working time all vehicles in a fleet is considered. Commonly, the daily working time of any vehicle should not longer than $8 \mathrm{hr}$. Therefore, $T_{\max }=8 \mathrm{hr}$. is set as the maximum working time in 
(13). The average speed of vehicles is $80 \mathrm{Km} / \mathrm{hr}$., approximately. Thus, the overall distance of each vehicle should not longer than $640 \mathrm{Km} /$ day. These data are utilized to define the number of vehicles $m$ in a fleet for each MVRP problem.

For comparison, searching parameters of GA, TS, PSO, FPA and MoFPA algorithms are set from recommendations and preliminary studies as follows: (i) for GA, number of offspring $=50$, percentage of crossover $=80 \%$ and percentage of mutation $=20 \%$, (ii) for TS, number of neighborhoods $=50$ and percentage of search radius $=20 \%$, (iii) for PSO, number of particles $=50$, cognitive learning rate $=$ 2.0 , social learning rate $=2.0$, inertia weight $\theta_{\min }=$ 0.4 and $\theta_{\max }=0.9$, (iv) for FPA, number of flowers $n$ $=50$ and switching probability $p=0.2$ (fixed) and (v) for MoFPA, number of flowers $n=50$ and randomly switching probability randp $\in[0.50,0.75]$ (random). The maximum iteration (Max_Iter = 10,000 ) is set as the termination criteria for all algorithms.

The experimental tests consist of two cases. For the first case, the central city of each MVRP problem will be set as the home city. For the second case, the corner-end city of each MVRP problem will be set as the home city.

\subsection{Case-I}

Once setting the central city as the home city, results of MVRP problem optimization obtained by GA, TS, PSO, FPA and MoFPA algorithms are summarized in Table 2. In this case, the convergent rates of the MVRP\#1 (Eil101) by the MoFPA algorithm over 50 trial-runs are plotted in Fig. 5 as an example. Each curve line in Fig. 5 represents the convergent rate of each trial-run. In addition, the optimal tours of all vehicles in a fleet of the MVRP\#1 (Eil101) obtained by GA, TS, PSO, FPA and MoFPA algorithms are displayed in Fig. 6-10, where a stands for the home city.

\subsection{Case-II}

When the corner-end city is set as the home city, results of MVRP problem optimization obtained by GA, TS, PSO, FPA and MoFPA are summarized in Table 3. For the second case, the convergent rates of the MVRP\#1 (Eil101) by the MoFPA algorithm over 50 trial-runs are plotted in Fig. 11 as an example. Each curve line in Fig. 11 represents the convergent rate of each trial-run. The optimal tours of all vehicles in a fleet of the MVRP\#1 (Eil101) obtained by GA, TS, PSO, FPA and MoFPA are displayed in Fig. 1216 , respectively.

Table 2 Optimal solutions of ten MVRP problems by GA, TS, PSO, FPA and MoFPA (Case-I).

\begin{tabular}{|c|c|c|c|c|c|c|c|c|c|c|c|}
\hline \multirow{2}{*}{ Problems } & \multirow{2}{*}{$\begin{array}{c}\text { No. of } \\
\text { Vehicle } \\
m\end{array}$} & \multicolumn{5}{|c|}{ Optimal Tour (Km.) } & \multicolumn{5}{|c|}{ Execution Time (sec.) } \\
\hline & & GA & TS & PSO & FPA & MoFPA & GA & TS & PSO & FPA & MoFPA \\
\hline MVRP\#1 & 5 & 765.99 & 761.72 & 738.90 & 733.46 & 724.66 & 112.89 & 91.15 & 57.63 & 35.09 & 35.08 \\
\hline MVRP\#2 & 8 & $16,022.36$ & $14,755.90$ & $12,427.25$ & $11,466.73$ & $10,912.64$ & 144.61 & 106.79 & 76.20 & 41.85 & 41.83 \\
\hline MVRP\#3 & 10 & $2,802.85$ & $2,650.48$ & $2,511.26$ & $2,476.12$ & $2,406.95$ & 225.33 & 154.66 & 102.65 & 67.22 & 67.19 \\
\hline MVRP\#4 & 11 & $38,994.21$ & $37,325.68$ & $36,986.03$ & $35,128.87$ & $34,358.26$ & 236.12 & 176.28 & 135.98 & 71.68 & 71.66 \\
\hline MVRP\#5 & 15 & $3,363.03$ & $3,295.83$ & $3,102.64$ & $2,898.57$ & $2,876.40$ & 258.37 & 198.62 & 150.23 & 80.29 & 80.26 \\
\hline MVRP\#6 & 17 & $65,035.74$ & $64,690.15$ & $63,672.71$ & $62,989.32$ & 62,576.08 & 341.83 & 256.10 & 198.41 & 105.63 & 105.65 \\
\hline MVRP\#7 & 24 & $311,585.18$ & $298,705.84$ & $279,664.02$ & $258,303.63$ & 254,934.07 & 439.66 & 344.58 & 215.86 & 136.35 & 136.31 \\
\hline MVRP\#8 & 25 & $73,980.55$ & $73,104.66$ & $72,602.71$ & $71,556.08$ & $71,120.43$ & 466.50 & 362.47 & 256.34 & 144.07 & 144.01 \\
\hline MVRP\#9 & 28 & $148,690.42$ & $146,159.07$ & $144,024.53$ & $142,606.71$ & $141,534.29$ & 557.61 & 443.42 & 312.40 & 173.32 & 173.38 \\
\hline MVRP\#10 & 30 & $3,508.97$ & $3,362.10$ & $3,188.73$ & $3,016.46$ & $2,955.02$ & 589.81 & 467.05 & 346.82 & 182.80 & 182.84 \\
\hline
\end{tabular}

Table 3 Optimal solutions of ten MVRP problems by GA, TS, PSO, FPA and MoFPA (Case-II).

\begin{tabular}{|c|c|c|c|c|c|c|c|c|c|c|c|}
\hline \multirow{2}{*}{ Problems } & \multirow{2}{*}{$\begin{array}{c}\text { No. of } \\
\text { Vehicle } \\
m\end{array}$} & \multicolumn{5}{|c|}{ Optimal Tour (Km.) } & \multicolumn{5}{|c|}{ Execution Time (sec.) } \\
\hline & & GA & TS & PSO & FPA & MoFPA & GA & TS & PSO & FPA & MoFPA \\
\hline MVRP\#1 & 5 & 908.83 & 899.94 & 893.85 & 890.02 & 886.86 & 112.92 & 91.12 & 57.65 & 35.04 & 35.10 \\
\hline MVRP\#2 & 8 & $17,488.29$ & $15,084.12$ & $13,936.77$ & $12,529.65$ & $11,562.34$ & 144.58 & 106.83 & 76.18 & 41.86 & 41.84 \\
\hline MVRP\#3 & 10 & $2,978.56$ & $2,797.53$ & $2,687.74$ & $2,615.31$ & $2,571.56$ & 225.36 & 154.61 & 102.60 & 67.13 & 67.19 \\
\hline MVRP\#4 & 11 & $40,126.44$ & $38,981.70$ & $38,012.68$ & $36,412.66$ & $35,503.78$ & 236.07 & 176.24 & 135.78 & 71.65 & 71.68 \\
\hline MVRP\#5 & 15 & $3,550.05$ & $3,401.46$ & $3,258.71$ & $3,101.88$ & $2,994.07$ & 258.32 & 198.59 & 150.26 & 80.31 & 80.27 \\
\hline MVRP\#6 & 17 & $66,912,42$ & $65,887.24$ & $64,925.63$ & $64,022.97$ & $63,876.51$ & 341.85 & 256.16 & 198.47 & 105.62 & 105.65 \\
\hline MVRP\#7 & 24 & $318,707.66$ & $310,886.33$ & $291,320.09$ & $262,515.80$ & $259,458.03$ & 439.69 & 344.45 & 215.83 & 136.37 & 136.32 \\
\hline MVRP\#8 & 25 & $75,607.12$ & $74,550.20$ & $73,904.48$ & $72,105.75$ & $71,988.62$ & 466.45 & 362.50 & 256.38 & 144.06 & 144.03 \\
\hline MVRP\#9 & 28 & $149,010.53$ & $147,088.46$ & $144,988.71$ & $144,090.60$ & $143,612.55$ & 557.67 & 443.48 & 312.47 & 173.31 & 173.39 \\
\hline MVRP\#10 & 30 & $3,616.04$ & $3,586.83$ & $3,311.64$ & $3,206.75$ & $3,164.30$ & 589.86 & 467.12 & 346.80 & 182.95 & 182.90 \\
\hline
\end{tabular}




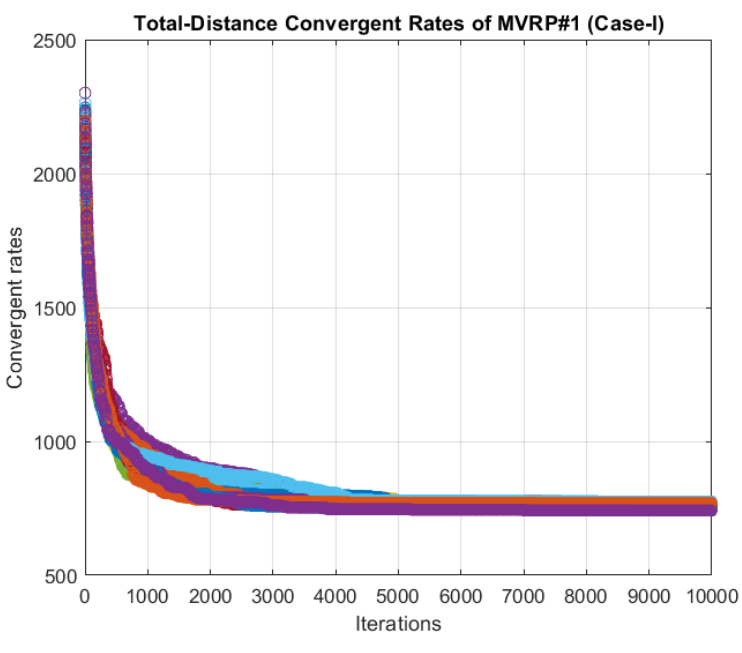

Fig. 5 Convergent rates by MoFPA (case-I).

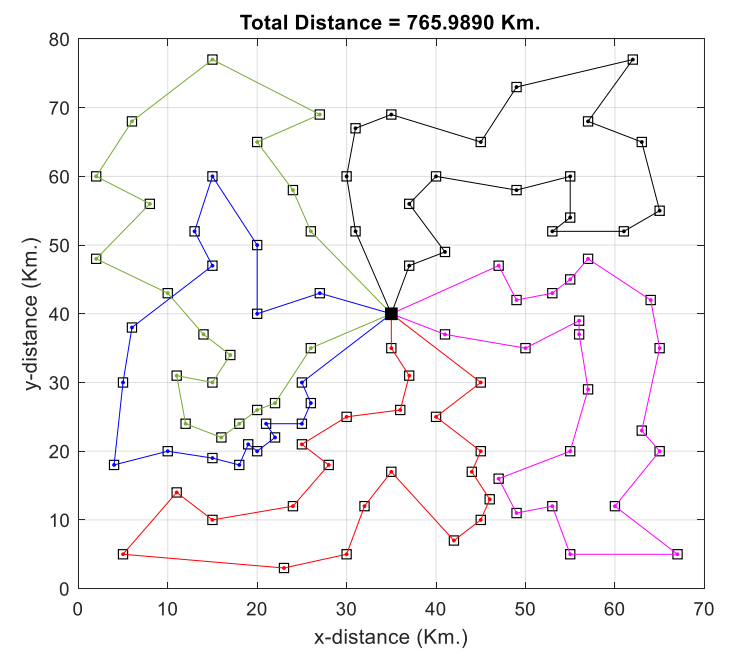

Fig. 6 Optimal tour of MVRP\#1 by GA (case-I).

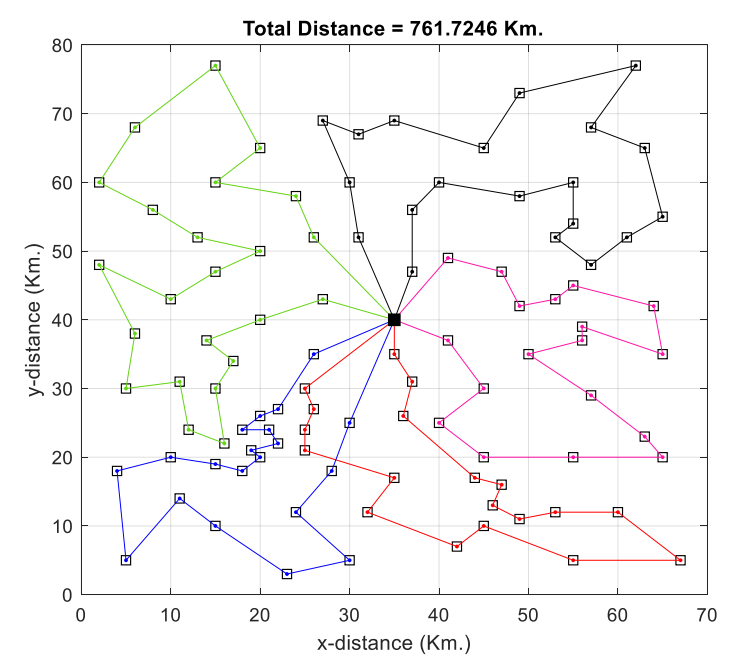

Fig. 7 Optimal tour of MVRP\#1 by TS (case-I).

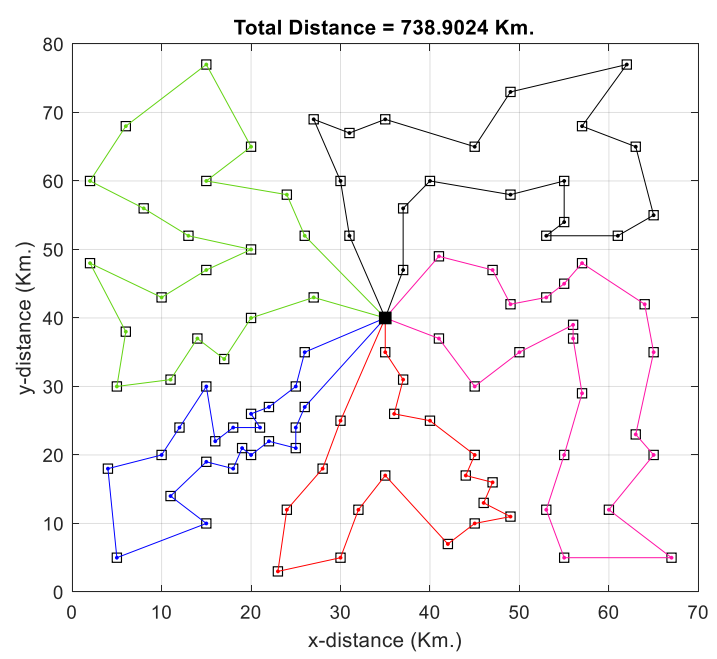

Fig. 8 Optimal tour of MVRP\#1 by PSO (case-I).

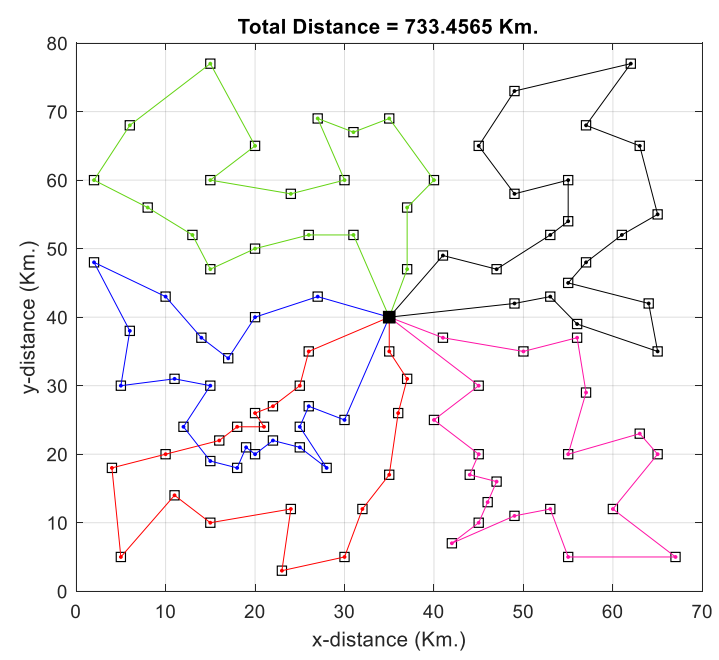

Fig. 9 Optimal tour of MVRP\#1 by FPA (case-I).

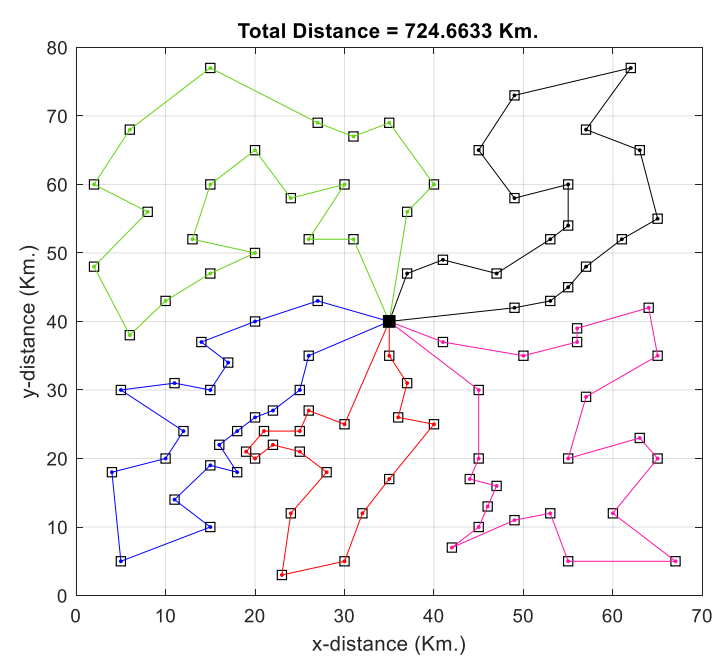

Fig. 10 Optimal tour of MVRP\#1 by MoFPA (case-I). 


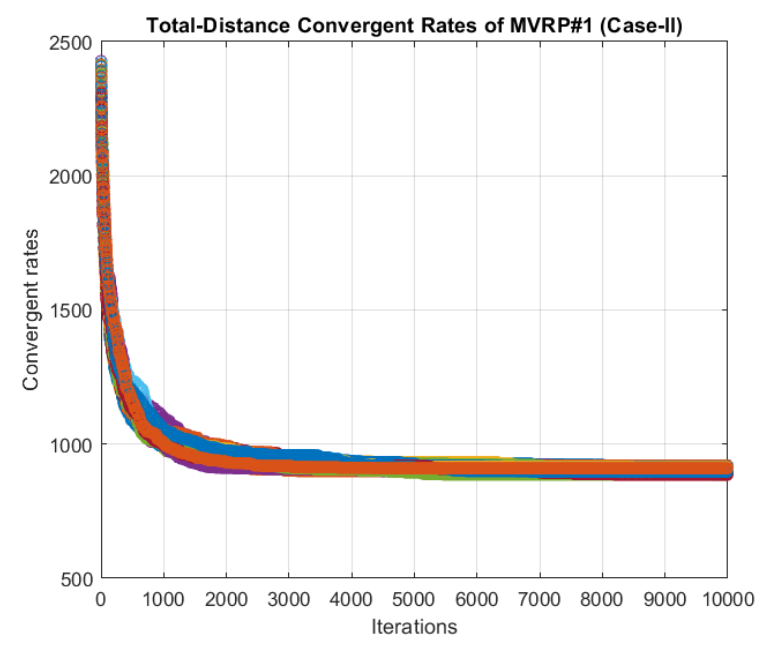

Fig. 11 Convergent rates of by MoFPA (case-II).

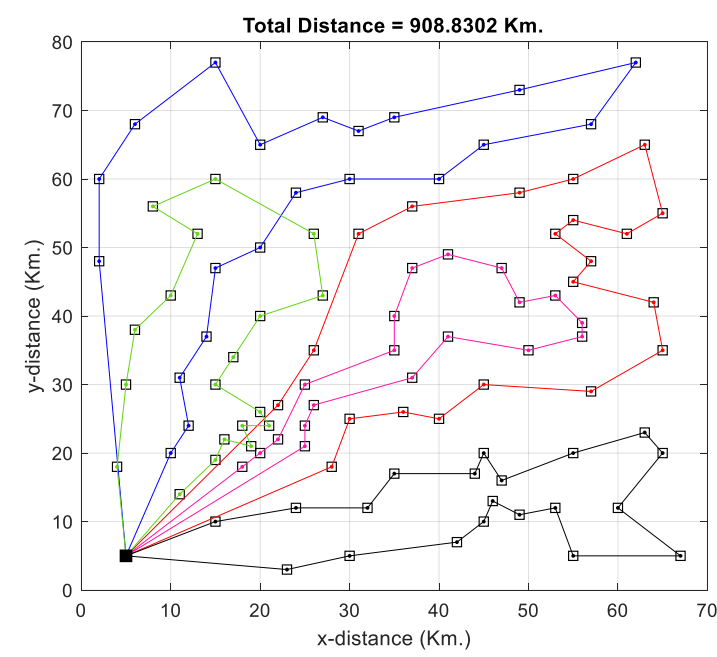

Fig. 12 Optimal tour of MVRP\#1 by GA (case-II).

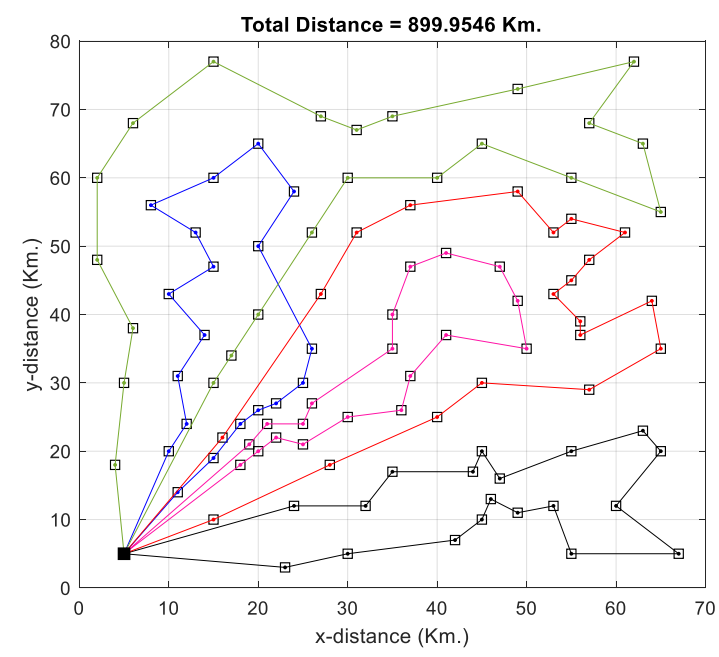

Fig. 13 Optimal tour of MVRP\#1 by TS (case-II).

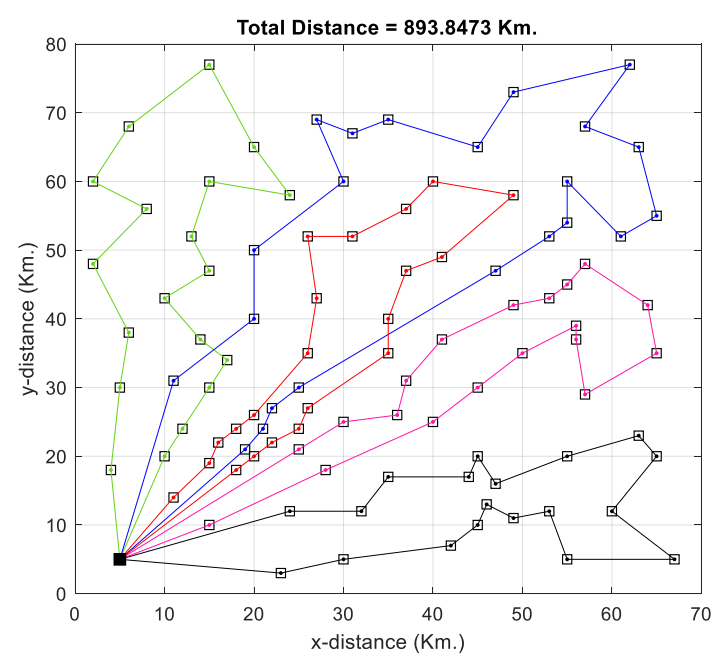

Fig. 14 Optimal tour of MVRP\#1 by PSO (case-II).

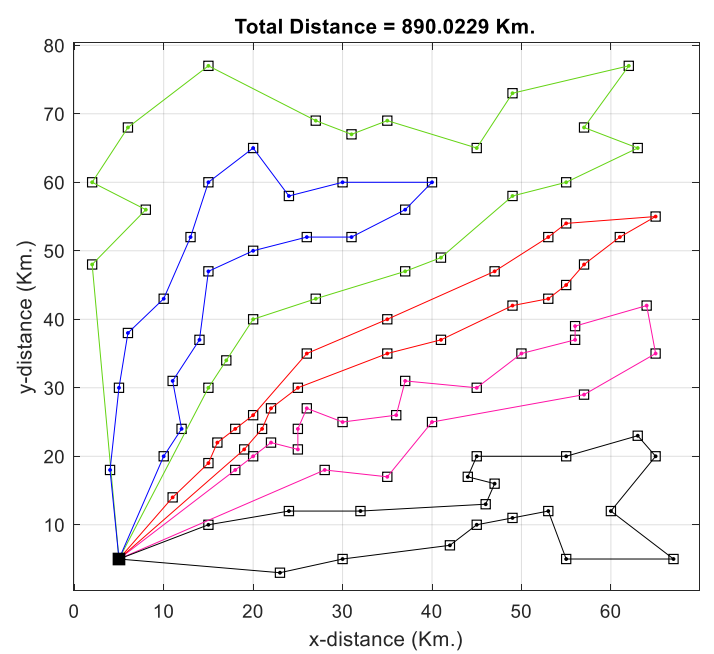

Fig. 15 Optimal tour of MVRP\#1 by FPA (case-II).

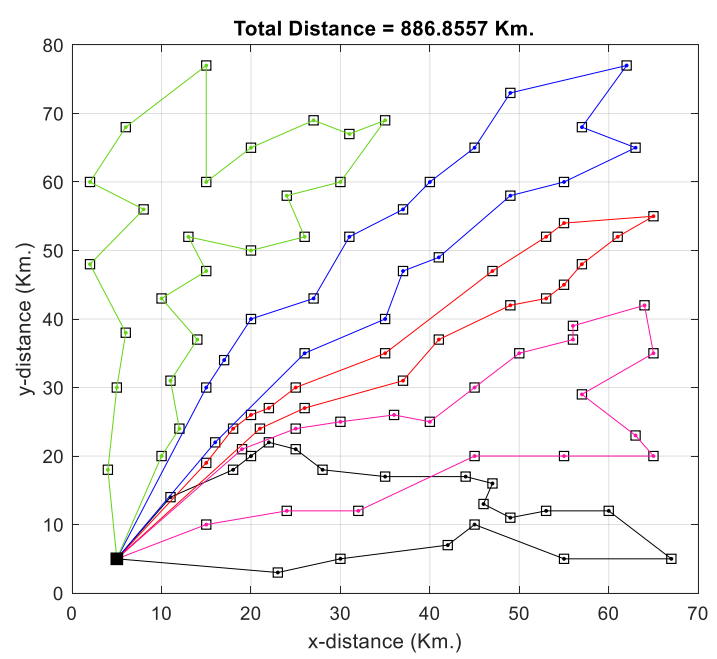

Fig. 16 Optimal tour of MVRP\#1 by MoFPA (case-II). 


\section{Discussions}

From the experimental results of case-I in section 4.1, it was found from Table 2 and Fig. 6-10 that the TS can provide shorter total distance than the GA for all ten MVRP problems with time constraints. The PSO can yield shorter total distance than the TS. The FPA can give shorter total distance than the PSO, while the MoFPA can provide shorter total distance than the FPA for all selected MVRP problems. Once considering the execution time, it can be observed that the TS consumes less execution time than the GA. The PSO consumes less execution time than the TS, while the FPA and MoFPA consume almost the same execution time which is less than the PSO. In addition, the execution time for searching the optimal solution (total distance) of all algorithms depends on the number of cities of the problem of interest. The more the number of cities, the more the execution time of all algorithms.

For the experimental results of case-II in section 4.2, it was found in Table 3 and Fig.12-16 that the TS can provide shorter total distance than the GA for all selected MVRP problems. The PSO can give shorter total distance than the TS. The FPA can yield shorter total distance than the PSO, while the MoFPA can provide shorter total distance than the FPA for all selected MVRP problems. When considering the execution time, it was found that the TS consumes less execution time than the GA. The PSO consumes less execution time than the TS, while the FPA and MoFPA consume almost the same execution time which is less than the PSO. Like case-I, the execution time for searching the optimal solution depends on the number of cities of the problem of interest. The more the number of cities, the more the execution time of all algorithms.

By comparison between case-I in Table 2 and case-II in Table 3, it can be noticed that the total distance of the MVRP problems in which the central city is set as the home city is shorter than that of the MVRP problems in which the corner-end city is set as the home city. From entire results, the MoFPA performs the superiority to other algorithms by providing the shorter total distance than FPA, PSO, TS and GA, respectively. The execution times of the FPA and MoFPA are almost the same, but they are less than that of PSO, TS and GA, respectively. Some relevant studies can be found $[25,26]$.

\section{Conclusions}

The application of the modified flower pollination algorithm or MoFPA to the MVRP problems with time constraints has been proposed in this paper. The
MoFPA, one of the latest enhanced versions of the original FPA utilizing the randomly switching probability for selection between local and global pollinations, has been applied to solve ten standard MVRP problems with time constraints consisting of approximately 100-500 destinations. Results obtained by the MoFPA have been compared with those obtained by GA, TS, PSO and original FPA. As experimental results, the MoFPA could provide optimal solutions of all ten selected MVRP problems with shorter total distance than FPA, PSO, TS and GA, respectively. The execution times of FPA and MoFPA are almost the same, but they are less than that of PSO, TS and GA, respectively. The execution time of all algorithms is increased, once the number of cities is increased. This confirms that the MVRP problem is in the class of NP-hard problems. In addition, the total distance of the MVRP problems in which the central city was set as the home city is shorter than that of the MVRP problems in which the corner-end city was set as the home city. The contribution and usefulness of this study can be applied in practical applicability for other real-world MVRP problems. For future research, the MoFPA will be applied to solve the vehicle routing balancing problems (VRBP) for balancing the working time, number of cities and distance of each vehicle in the fleet. The multiple-depots multiple-vehicle routing problems (MD-MVRP) will be also investigated by MoFPA and other novel metaheuristic optimization search techniques.

\section{Acknowledgements}

This research was funded by King Mongkut's University of Technology North Bangkok, Thailand. Contract no. KMUTNB-63-DRIVE-8.

\section{References:}

[1] X. S. Yang, Flower Pollination Algorithm for Global Optimization, Unconventional Computation and Natural Computation, Lecture Notes in Computer Science, Vol. 7445, 2012, pp. 240249.

[2] X. S. He, X. S. Yang, M. Karamanoglu and Y. $X$. Zhao, Global Convergence Analysis of the Flower Pollination Algorithm: a Discrete-Time Markov Chain Approach, Procedia Computer Science, Vol. 108, 2017, pp. 1354-1364.

[3] X. S. Yang, M. Karamanoglua and X. He, MultiObjective Flower Algorithm for Optimization, Procedia Computer Science, Vol. 18, 2013, pp. 861-868. 
[4] H. Chiroma, N. L. M. Shuib, S. A. Muaz, A. I. Abubakar, L. B. Ila and J. Z. Maitama, A Review of the Applications of Bio-Inspired Flower Pollination Algorithm, Procedia Computer Science, Vol. 62, 2015, pp. 435-441.

[5] Z. A. A. Alyasseri, A. T. Khader, M. A. AlBetar, M. A. Awadallah and X. S. Yang, Variants of the Flower Pollination Algorithm: a Review, Nature-Inspired Algorithms and Applied Optimization: Studies in Computational Intelligence, Vol. 744, 2018, pp. 91-118.

[6] E. Nabil, A Modified Flower Pollination Algorithm for Global Optimization, Expert Systems with Applications, Vol. 57, 2016, pp. 192-203.

[7] D. Rodrigues, X. S. Yang, A. N. De Souza, and J. P. Papa, Binary Flower Pollination Algorithm and Its Application to Feature Selection, Recent Advances in Swarm Intelligence and Evolutionary Computation, Springer, 2015, pp. 85-100.

[8] M. Y. Shambour, A. A. Abusnaina, and A. I. Alsalibi, Modified Global Flower Pollination Algorithm and Its Application for Optimization Problems, Interdisciplinary Sciences: Computational Life Sciences, Springer, 2018, pp.1-12.

[9] N. Pringsakul and D. Puangdownreong, Modified Flower Pollination Algorithm for Function Optimization, The International Conference on Intelligent Computing and Optimization (ICO'2020), 2020.

[10] N. Pringsakul and D. Puangdownreong, MoFPA-Based PIDA Controller Design Optimization for Electric Furnace Temperature Control System, International Journal on Electrical Engineering and Informatics, VOl. 16, No. 6, 2020. (In-print).

[11] B. J. Glover, Understanding Flowers and Flowering: An Integrated Approach, Oxford University Press, 2007.

[12] P. Willmer, Pollination and Floral Ecology, Princeton University Press, 2011.

[13] K. Balasubramani and K. Marcus, A Study on Flower Pollination Algorithm and Its Applications, International Journal of Application or Innovation in Engineering \& Management (IJAIEM), Vol. 3, 2014, pp. 320325.

[14] T. Bektas, The Multiple Traveling Salesman Problem: an Overview of Formulations and Solution Procedures, Omega, Vol. 34, No. 3, 2006, pp. 209-219.
[15] M. Bellmore and G. L. Nemhauser, The Traveling Salesman Problem: A Survey, Operation Research, Vol. 16, 1986, pp. 538558.

[16] H. Fleishner, Traversing graphs: The Eulerian and Hamiltonian theme, M. Dror (ed.), Arc Routing: Theory, Solutions, and Applications, Kluwer Acad. Publ., 2000.

[17] V. Ungureanu, Traveling Salesman Problem with Transportation, Computer Science Journal of Moldova, Vol. 14, No. 2(41), 2006, pp. 202206.

[18] S. Kikuchi and P. Chakroborty, Place of Possibility Theory in Transportation Analysis, Transportation Research, Part B, Vol. 40, 2006, pp. 595-615.

[19] B. L. Golden and A. A. Assad, Vehicle Routing: Methods and Studies, Elsevier Science, Amsterdam, 1988.

[20] X. C. Shi, Y. C. Liang, H. P. Lee, C. Lu and Q. X. Wang, Particle Swarm Optimization-Based Algorithms for TSP and Generalized TSP, Information Processing Letters, Vol. 103, 2007, pp. 169-176.

[21] S. Suwannarongsri and D. Puangdounreong, Optimal Solving Large Scale Traveling Transportation Problems by Flower Pollination Algorithm, WSEAS Transactions on Systems and Control, Vol. 14, 2019, pp. 19-24.

[22] S. Suwannarongsri, Solving Multiple Vehicle Routing Problems with Time Constraints by Flower Pollination Algorithm Optimization, WSEAS Transactions on Systems, Vol. 19, 2020, pp. 178-187.

[23] TSPLIB95, Symmetric traveling salesman problem, http://comopt.ifi.uniheidelberg.de/ software/ TSPLIB95/, 2011.

[24] M. Held and R. Karp, A Dynamic Programming Approach to Sequencing Problems, SIAM Journal, Vol. 1, 1962, pp. 196-210.

[25] T. Jitwang, A. Nawikavatan and D. Puangdownreong, Optimal PIDA Controller Design for Three-Tank Liquid-Level Control System with Model Uncertainty by Cuckoo Search, International Journal of Circuits, Systems and Signal Processing, Vol. 13, 2019, pp. 60-65.

[26] S. Semami, H. Toulni and A. ElByed, The Close Enough Traveling Salesman Problem with Time Window, International Journal of Circuits, Systems and Signal Processing, Vol. 13, 2019, pp. 579-584.

Creative Commons Attribution License 4.0 (Attribution 4.0 International, CC BY 4.0)

This article is published under the terms of the Creative Commons Attribution License 4.0

https://creativecommons.org/licenses/by/4.0/deed.en US 